\title{
Application of Fuzzy AR (p) Model in Data Processing of Deformation Monitoring
}

\author{
Wei Chen ${ }^{1, a}$,Jian Zhang ${ }^{2, b}$ \\ ${ }^{1}$ College of Urban Construction,Wuhan University of Science and Technology, China \\ 2 Wuhan KQ GEO Instruments Co., Ltd, China \\ anancychw@163.com, bzhenhuyao@163.com
}

\begin{abstract}
Keywords: fuzzy $\operatorname{AR}(p)$ model;fuzzy number;deformation monitoring;data processing
Abstract. Compared with general time series forecasting methods, fuzzy time series has the main advantage of dealing with linguistic variables or fuzzy data in time series. In this paper, the fuzzy AR (p) model estimation theory is introduced into the data processing of deformation monitoring,and the steps of modeling fuzzy AR (p) model are briefly given. Finally, an example is given to prove that it is effective and feasible to apply fuzzy AR (p) model to the data processing of deformation monitoring.
\end{abstract}

\section{Introduction}

At present many prediction methods are modeled based on observation data that are accurate values,but many observation data or statistical data obtained in practical work often have the characteristic of fuzziness. These fuzzy phenomena can not been effectively described and dealt with by classical methods of probability theory. In the field of surveying data processing, parameter estimation theory, such as least square estimation, treats measurement errors as random variables. In fact the uncertainty of measurement data is not only random, but also fuzzy. These phenomena which are not only random but also fuzzy can be predicted by fuzzy time series.

Compared with general time series forecasting methods, fuzzy time series has the main advantage of dealing with linguistic variables or fuzzy data in time series.Considering that the uncertainty of measurement data is not only random, but also fuzzy, the fuzzy AR (p) model estimation theory will be applied to the data processing of deformation monitoring, and the example will be given to prove its feasibility and effectiveness.

\section{Fuzzy AR (p) Prediction Model}

Related definitions. Supposing that $\left\{\tilde{x}_{t}\right\}$ is stationary fuzzy random time series, if $\left\{\tilde{x}_{t}\right\}$ satisfies

$$
\tilde{x}_{t}=\varphi_{1} \tilde{x}_{t-1}+\varphi_{2} \tilde{x}_{t-2}+\mathrm{L}+\varphi_{p} \tilde{x}_{t-p}+\tilde{a}_{t}
$$

$\left\{\tilde{x}_{t}\right\}$ is called p order fuzzy autoregressive time series,and the corresponding model is called fuzzy $\mathrm{AR}(\mathrm{p})$ model, denoted as FAR(p) model. Where $\tilde{x}_{t}=\left(x_{t}, \delta_{t}\right)$ is a fuzzy variable $\left(x_{t}\right.$ is the fuzzy center of $\tilde{x}_{t}$, and $\delta_{t}$ is the fuzzy range), $\varphi_{i}(\mathrm{i}=1,2, \ldots \mathrm{p})$ is the fuzzy autoregressive parameter and $\tilde{a}_{t}$ is fuzzy white noise.

If the observation data themselves are fuzzy numbers, these data can be modeled directly. If the observation data is fuzzy numbers, but only a series of figures $x_{1}, x_{2}, \ldots, x_{N}$ which are actually the exact numbers of fuzzy numbers are recorded in the actual data collection process. In this case, before modeling, we must construct a set of fuzzy numbers according to the exact values obtained, and then establish the model. The triangular fuzzy numbers will be constructed in this paper.

The method of constructing triangular fuzzy numbers is as follows:

Assume that the data has been collected in chronological order as $x_{t}(t=1,2, \mathrm{~L}, N)$, we define: 


$$
\begin{aligned}
& U_{t}=\max \left(x_{t-1}, x_{t}, x_{t+1}\right),(t=2,3, \mathrm{~L}, N-1) \\
& V_{t}=\min \left(x_{t-1}, x_{t}, x_{t+1}\right),(t=2,3, \mathrm{~L}, N-1) \\
& U_{1}=\max \left(x_{1}, x_{2}\right) ; V_{1}=\min \left(x_{1}, x_{2}\right) ; U_{N}=\max \left(x_{N-1}, x_{N}\right) ; V_{N}=\min \left(x_{N-1}, x_{N}\right) \\
& x_{t}=\frac{1}{2}\left(U_{t}+V_{t}\right),(t=1,2, \mathrm{~L}, N) \\
& \delta_{t}=\frac{1}{2}\left(U_{t}-V_{t}\right),(t=1,2, \mathrm{~L}, N)
\end{aligned}
$$

Thus, the original time series $\left\{x_{t}\right\}$ is transformed into a fuzzy time series $\left\{\tilde{x}_{t}\right\}$

$$
\tilde{x}_{t}=\left(x_{t}, \delta_{t}\right),(t=1,2, \mathrm{~L}, N)
$$

The model (1) can be determined by model identification, parameter estimation and order determination. Model identification is determined by the fuzzy partial correlation function of the sample; the model parameters are determined by using fuzzy least squares estimation;model order is determined by F-test rank determination method.

Parameter estimation of fuzzy AR(p) model. Assuming that the order $p$ of the model (1) has been determined, how to determine the autoregressive coefficients $\varphi_{1}, \varphi_{2}, \ldots, \varphi_{p}$ of model (1) will be given. In this paper, the fuzzy least square estimation method is used to determine the model parameters. For an observation sequence $\left\{\tilde{x}_{t}\right\}$, let $\hat{x}_{t}$ be the fuzzy estimated value of $\tilde{x}_{t}$, then

$$
\hat{x}_{t}=\hat{\varphi}_{1} \tilde{x}_{t-1}+\hat{\varphi}_{2} \tilde{x}_{t-2}+\mathrm{L}+\hat{\varphi}_{p} \tilde{x}_{t-p}
$$

According to the operation rules of fuzzy numbers, we can get $\hat{x}_{t}=\left(\sum_{i=1}^{p} \hat{\varphi}_{i} x_{t-i}, \sum_{i=1}^{p} \hat{\varphi}_{i} \delta_{t-i}\right)$. Then the fuzzy distance between the fuzzy number $\tilde{x}_{t}$ and the estimated value $\hat{x}_{t}$ is:

$$
D\left(\tilde{x}_{t}, \hat{x}_{t}\right)=\left(x_{t}-\sum_{i=1}^{p} \hat{\varphi}_{i} x_{t-i}\right)^{2}+\left(\delta_{t}-\sum_{i=1}^{p} \hat{\varphi}_{i} \delta_{t-i}\right)^{2}
$$

The function of the sum of squares of the fuzzy distance is described as:

$$
\varphi(D)=\sum_{t=p+1}^{N} D\left(\tilde{x}_{t}, \hat{x}_{t}\right)=\sum_{t=p+1}^{N}\left\{\left(x_{t}-\sum_{i=1}^{p} \hat{\varphi}_{i} x_{t-i}\right)^{2}+\left(\delta_{t}-\sum_{i=1}^{p} \hat{\varphi}_{i} \delta_{t-i}\right)^{2}\right\}
$$

We use the FAR(p) model to predict, and the evaluation criterion is that the smaller the distance between the fuzzy number $\tilde{x}_{t}$ and the estimated value $\hat{x}_{t}$ is, the better the estimation precision is,that is

$$
\varphi(D)=\min
$$

Therefore, according to formula (5), the normal equations can be obtained by taking the partial derivatives of the parameters $\hat{\varphi}_{1}, \hat{\varphi}_{2}, \mathrm{~L}, \hat{\varphi}_{p}$.

$$
\frac{\partial \varphi(D)}{\partial \varphi_{j}}=0(j=1,2, \mathrm{~L}, p)
$$


Then the estimated values $\hat{\varphi}_{1}, \hat{\varphi}_{2}, \mathrm{~L}, \hat{\varphi}_{p}$ of $\operatorname{FAR}(\mathrm{p})$ model parameters $\varphi_{1}, \varphi_{2}, \ldots, \varphi_{p}$ can be obtained by solving the system of equations.

\section{Application in the data processing of deformation monitoring}

In this section,the fuzzy AR(p) model is applied to data processing of deformation monitoring. The data of the example come from the observation data of a pier in evaluation section of settlement observation project of Wuhan-Xianning high speed railway.We adopt the first forty period data to establish the fuzzy AR(p) model and predict the latter five period data. The predicted value is compared with the measured value to verify the accuracy and reliability of the prediction.

The criterion for evaluating the forecast results in the paper is as follows:

(1) Mean Square Error $(M S E): \quad M S E=\frac{1}{n} \sum_{t=1}^{n}\left(y_{t}-\hat{y}_{t}\right)^{2}$

(2) Mean Absolute Percentage Error (MAPE ): $M A P E=\frac{1}{n} \sum_{t=1}^{n} \frac{\left|y_{t}-\hat{y}_{t}\right|}{\left|y_{t}\right|} \times 100$

Where $y_{t}$ are Observations, $\hat{y}_{t}$ are predicted values and $n$ is the number of observations.

According to the fuzzy $\operatorname{AR}(\mathrm{p})$ modeling process introduced in this paper, the model parameters are determined by using fuzzy least squares estimation;model order is determined by F-test rank determination method. The parameter estimation and the sum of squares of the fuzzy distance for each order of fuzzy AR model are shown in table 1.

Table 1 Parameter estimation and the sum of squares of the fuzzy distance

\begin{tabular}{c|c|c|c}
\hline & $\varphi_{1}$ & $\varphi_{2}$ & $\varphi(\mathrm{D})$ \\
\hline $\operatorname{FAR}(1)$ & -0.45541 & & 7.112 \\
\hline FAR(2) & 1.3644 & -0.0702 & 33.476 \\
\hline
\end{tabular}

According to the F-test rank determination method,the condition $F<F_{\alpha}$ is satisfied when the order is equal to 1 . Therefore, the model order is 1 , and the model is described as FAR(1).The fuzzy $\mathrm{AR}(\mathrm{p})$ model in this example is described as follows:

$$
\tilde{x}_{t}=-0.45541 \tilde{x}_{t-1}+\tilde{a}_{t}
$$

Thus the final prediction formula is obtained as follows:

$$
x_{t}=1.54459 x_{t-1}-0.08918 x_{t-2}-0.45541 x_{t-3}
$$

According to the formula (9), the latter five period data are predicted. For comparison,the AR(p) model and the fuzzy AR(p) model are separately established to predict the same data in this example,and the forecasted values and accuracy assessment results of two models are shown in table 2 .

As can be seen from table 2, the maximum error between the predicted values of the fuzzy AR (p) model and the original observations is $0.4 \mathrm{dmm}$, which meet the allowable error, and it can better reflect the trend of the settlement curve. The results in table 2 shows the superiority of the fuzzy AR(p) model over the $\mathrm{AR}(\mathrm{p})$ model as it provides forecast of higher accuracy. Also,experimental results show that it is feasible and effective to apply the fuzzy AR(p) model estimation theory to the data processing of deformation monitoring. 
Table 2 Prediction results of two models

\begin{tabular}{|c|c|c|c|c|c|}
\hline \multirow{2}{*}{$\begin{array}{c}\text { Number } \\
\text { of } \\
\text { Periods }\end{array}$} & \multirow{2}{*}{$\begin{array}{c}\text { Observed } \\
\text { Value } \\
(\mathrm{dmm})\end{array}$} & \multicolumn{2}{|c|}{ Least Square Estimation } & \multicolumn{2}{|c|}{$\begin{array}{c}\text { Fuzzy Least Square } \\
\text { Estimation }\end{array}$} \\
\hline & & $\begin{array}{c}\text { Predicted } \\
\text { Value } \\
(\mathrm{dmm}) \\
\end{array}$ & $\begin{array}{c}\text { relative } \\
\text { error } \\
(\%) \\
\end{array}$ & $\begin{array}{c}\text { Predicted } \\
\text { Value } \\
(\mathrm{dmm}) \\
\end{array}$ & $\begin{array}{c}\text { relative } \\
\text { error } \\
(\%) \\
\end{array}$ \\
\hline 41 & -20.6 & -20.3 & 1.46 & -20.5 & 0.48 \\
\hline 42 & -20.5 & -20.3 & 0.97 & -20.3 & 0.98 \\
\hline 43 & -20.5 & -20.0 & 2.44 & -20.2 & 1.46 \\
\hline 44 & -20.2 & -19.7 & 2.47 & -19.9 & 1.48 \\
\hline 45 & -20.1 & -19.4 & 3.48 & -19.7 & 1.99 \\
\hline \multicolumn{2}{|c|}{ MSE } & \multicolumn{2}{|c|}{0.224} & \multicolumn{2}{|c|}{0.078} \\
\hline \multicolumn{2}{|c|}{ MAPE } & \multicolumn{2}{|c|}{2.164} & \multicolumn{2}{|c|}{1.278} \\
\hline
\end{tabular}

\section{Conclusion}

Considering that the uncertainty of measurement data is not only random, but also fuzzy, the fuzzy AR (p) model estimation theory has been applied to the data processing of deformation monitoring. Experimental results show that it is feasible and effective to apply the fuzzy AR (p) model estimation theory to the data processing of deformation monitoring.How to more widely apply the fuzzy AR (p) model estimation theory to the data processing of deformation monitoring will need further study.

\section{References}

[1] Q. Song, B. Chissom:Fuzzy sets and Systems, Vol. 62 (1993) p.1-8.

[2] Hamilton J:Time Serie Analysis.(Princeton University Press,1994.)

[3] J. P. Wu:Systems Engineering, Vol. 20 (2002), p.72-76.

[4] D. Domanska,M. Wojtylak:Expert Systems with Application, Vol. 39 (2012) p. 7673-7679.

[5] C. Shen,Y.X. Sun:Journal of Computer Applicaions, Vol. 33 (2013) p. 1222-1224.

[6] W. Chen:Applied Mechanics and Materials, Vol. 405-408 (2013) p. 2448-2451.

[7] J. R. Wang,H. Wen,Z. Q. Luo:Coal, Vol. 6 (2015), p.3-6. 